\title{
Diversidade de Gênero e Evasão Universitária nos Cursos de Engenharia
}

\author{
DOI: 10.37702/2175-957X.COBENGE.2021.3516
}

Tifane de Paula Carvalho - tifane.paulacarvalho@gmail.com

Universidade Federal de Uberlândia

RUA FRANCISCO RODRIGUES SILVA 1283

12236-460 - SÃO JOSÉ DOS CAMPOS - SP

Ana Marta Souza - anamartaengenharia@gmail.com

Universidade Federal de Uberlândia

Rua Jangadeiros 201

38412-016 - Uberlândia - MG

Ligia Carolina Oliveira Silva - ligiacarol@ufu.br

Universidade Federal de Uberlândia

Praça Alcides Borges de Oliveira 10

38408-142 - Uberlândia - MG

Resumo: $O$ presente estudo busca entender e quantificar o fenômeno da evasão em instituições do ensino superior, mais especificamente, das diferenças entre a evasão de mulheres e de homens nos cursos de Engenharia Mecânica, Engenharia Mecatrônica e Engenharia Aeronáutica da Universidade Federal de Uberlândia, através da validação da hipótese de que as mulheres apresentam maiores taxas de evasão em cursos de Engenharia, levando em consideração a diversidade do fator humano e as dificuldades enfrentadas por mulheres que optam em seguir carreiras STEM (Ciência, Tecnologia, Engenharia e Matemática). Os dados foram coletados a partir de relatórios estatísticos da Instituição de Ensino para os cursos de Engenharia Mecânica, Mecatrônica e Aeronáutica da Universidade Federal de Uberlândia entre os anos de 2010 a 2019. Os resultados obtidos a partir de análises qualitativas dos dados apontam uma maior evasão de discentes do sexo feminino. Enquanto apenas $24,17 \%$ dos homens matriculados nos três cursos evadiram, 29,65\% das mulheres o fizeram.

Palavras-chave: Evasão. STEM. Engenharia. Gênero. 


\section{DIVERSIDADE DE GÊNERO E EVASÃO UNIVERSITÁRIA NOS CURSOS DE ENGENHARIA}

\section{INTRODUÇÃO}

O conceito de evasão diverge segundo cada autor. Verifica-se a existência de diversas definições para este fenômeno e inúmeras metodologias para estudá-lo. Para os fins do presente artigo, optou-se por destacar a definição feita por Palharini (2010), para o mesmo, a evasão é a saída definitiva do aluno do curso de origem sem concluí-lo, sendo consideradas as seguintes formas de saída: não realização da matrícula e abandono do curso, comunicação de desistência oficialmente, realização de transferência para outro curso da mesma instituição, exclusão por norma institucional, ou ainda, transferência para outra instituição de ensino.

A importância de estudar o fenômeno da evasão justifica-se a partir da afirmação de Silva Filho et al (2007), segundo ele a evasão estudantil é um problema internacional, que afeta o resultado dos sistemas educacionais. As perdas de estudantes implicam desperdícios sociais, acadêmicos e econômicos. Em concordância tem-se Prestes, Fialho e Pfeiffer (2014) que também indicam perdas sociais, econômicas, além do descumprimento com os objetivos institucionais na formação de cidadão, como consequências da evasão.

Segundo dados do Censo de Educação Superior de 2018, divulgados pelo Instituto Nacional de Estudos e Pesquisas Educacionais Anísio Teixeira (INEP), a evasão universitária apresenta uma taxa de $41 \%$. Com relação a cursos de engenharia, a porcentagem é ainda maior e, portanto, mais alarmante.

O problema da evasão pode ser estudado em termos das peculiaridades dos alunos evadidos. Pode-se analisar o problema segundo aspectos demográficos, sociais e relacionados a diversidade do fator humano. Levando-se em consideração que, segundo o INEP (Instituto Nacional de Estudos e Pesquisas Educacionais Anísio Teixeira), nos cursos de engenharia, as mulheres representam apenas $20,3 \%$ dos matriculados e que apenas $60 \%$ concluem o curso, porcentagens ainda menores a depender dos cursos de engenharia, assim, uma análise sobre a evasão por gênero torna-se pertinente e necessária. A falta de representatividade nos cursos de engenharia justifica-se diante da sociedade em que vivemos onde as mulheres não são incentivadas a se inscrever em cursos STEM, tal conceito refere-se ao ensino e aprendizado nos campos da ciência, tecnologia, engenharia e matemática. Normalmente inclui atividades educacionais em todos os níveis de ensino (Gonzalez e Kuenzi, 2012).

A UNESCO (Organização das Nações Unidas para a Educação a Ciência e a Cultura) desenvolveu o estudo: "Decifrar o Código: educação de meninas e mulheres em STEM" (2018) que aponta que a sub-representação de mulheres em STEM tem raízes profundas já que muitas meninas são impedidas de se desenvolver por conta da discriminação, pelos diversos vieses e normas, além de expectativas sociais que influenciam a qualidade da educação que as mesmas recebem e os assuntos que estudam. $\mathrm{O}$ estudo identifica quatro fatores principais que influenciam a participação, o avanço e o desempenho de meninas e mulheres na educação em STEM: 
- Âmbito individual: habilidades linguísticas e espaciais, interesse, envolvimento, motivação e satisfação, autoeficácia e autopercepção além dos estereótipos de identidade em STEM.

- Âmbitos familiar e de pares: crenças e expectativas dos pais, nível de instrução dos pais, status socioeconômico e outros fatores domésticos, assim como a influência dos pares.

- Âmbito escolar: fatores inerentes ao ambiente de aprendizagem, incluindo o perfil dos docentes, suas experiências, crenças e expectativas, os currículos, os materiais e recursos de aprendizagem, as estratégias de ensino, as interações estudante-docente, as práticas avaliativas e o ambiente escolar em geral.

- Âmbito social: normas sociais e culturais relacionadas à igualdade de gênero, e os estereótipos de gênero presentes na mídia.

Associada aos baixos números de mulheres em cursos de STEM, temos também a elevada dificuldade das mesmas de permanecerem nos cursos. Uma pesquisa desenvolvida na UFPB (Universidade Federal da Paraíba) com ex-alunas de Física mostra a existência de uma cultura masculina com práticas preconceituosas e discriminatórias, explícitas e implícitas. Segundo Pinto e Amorim (2015), as ex-alunas eram tratadas com frieza, estranhamento e até mesmo como objetos sexuais. Infelizmente tais práticas se estendem para outros cursos considerados tipicamente masculinos.

Outros estudos já foram desenvolvidos em relação à evasão por gênero em instituições do ensino superior. As conclusões são diversas. Quantitativamente, Stinebricker e Stinebricker (2013) e Bound, Lovenheim e Turner (2009) mostram que as mulheres abandonam menos os cursos. Já Smith e Naylor (2001) apresentam que as taxas são homogêneas. Silva (2011) conclui que as mulheres persistem menos do que os homens, com base em estudo realizado em uma instituição privada brasileira.

Poucos trabalhos, no entanto, analisam à evasão por gênero para cursos STEM. Apenas dois trabalhos com tais características foram identificados após exaustiva pesquisa. Isphording e Qendrai (2019) quantificaram as taxas de evasão por gênero em cursos de graduação, a partir de uma pesquisa representativa com estudantes da Alemanha. Os resultados apontaram uma significativa diferença nas taxas. As mulheres evadem mais em cursos de STEM, enquanto os homens evadem mais em cursos não relacionados às ciências exatas. Além disso, a evasão de mulheres em cursos de ciência, tecnologia, engenharia e matemática, são superiores a qualquer outra taxa, de homens e mulheres nos mais diversos cursos. Já Valdiero et al (2014) desenvolveram uma análise da evasão de acadêmicos do curso de Engenharia Mecânica da Universidade Regional do Noroeste do Estado do Rio Grande do Sul, com atenção especial para a situação do gênero. Para a coleta de informações, foram utilizados os laudos disponíveis no Sistema de Informações Educacionais da Instituição em relação ao período de 2007 a 2014. A partir da análise dos dados, concluiu-se que ocorreu uma evasão de $56 \%$ de acadêmicas do sexo feminino e de $56 \%$ de acadêmicos do sexo masculino. As mulheres se mostraram mais persistentes nos primeiros anos, mantendo uma porcentagem de cerca de $9 \%$ entre o total de acadêmicos com matrícula ativa. Entretanto, as mulheres representaram apenas $2 \%$ entre os formados até o final de 2013.

O presente trabalho visa então acessar uma área ainda incipiente sobre a evasão, coletando dados do evadidos dos cursos de engenharia da Universidade Federal de Uberlândia (UFU) de maneira a validar, ou não, a hipótese levantada de que há diferenças nas taxas de evasão de homens e mulheres, sendo que, as mulheres evadem mais. 


\section{PROCEDIMENTOS METODOLÓGICOS}

\subsection{Amostra}

O público alvo do presente estudo constituiu-se de discentes dos cursos de Engenharia Mecânica, Mecatrônica e Aeronáutica da Universidade Federal de Uberlândia, matriculados entre os anos de 2010 e 2019. Foram computados 2000 discentes matriculados no período, sendo apenas 297 do sexo feminino. Os números de evadidos também foram computados, para o mesmo período, em que 423 discentes do sexo masculino e 86 do sexo feminino evadiram. Na tabela abaixo é possível observar os números de matriculados em cada curso por gênero, assim como a quantidade de evadidos, também por gênero.

Tabela 1: Matriculados e evadidos por curso e por gênero no período 2010-2019

\begin{tabular}{lcccc}
\hline \multicolumn{1}{c}{ Curso } & \multicolumn{2}{c}{ Matriculados } & \multicolumn{2}{c}{ Evadidos } \\
\hline & Homens & Mulheres & Homens & Mulheres \\
\hline Engenharia Mecânica & 903 & 119 & 221 & 39 \\
\hline Engenharia Mecatrônica & 376 & 105 & 92 & 29 \\
\hline Engenharia Aeronáutica & 434 & 63 & 110 & 18 \\
\hline
\end{tabular}

Fonte: Elaborada pelo autor.

\subsection{Instrumentos}

Os dados necessários para o desenvolvimento do presente trabalho foram obtidos através dos relatórios estatísticos da Instituição para os cursos analisados. A partir de tais dados, foi possível determinar a taxa de evasão por gênero, para os cursos analisados a. Para o tratamento dos dados, utilizou-se o software Excel.

\subsection{Procedimentos}

Primeiramente, os dados foram coletados a partir de relatórios gerados pela Instituição entre os anos de 2010 e 2019 para cada um dos cursos. Em seguida, os dados foram transferidos para o Software Excel, os ingressantes foram separados segundo 0 gênero, assim como os egressos. Foram consideradas às saídas por desistência oficial, transferência interna, abandono e jubilamento.

Os números de ingressantes, por gênero, para os cursos de Engenharia Mecânica, Mecatrônica e Aeronáutica foram contabilizados para o período analisado, assim como as taxas de evasão. As mesmas foram calculadas a partir da Equação 1.

$$
E_{v}=\frac{E_{p}}{M_{p}} * 100 \%
$$

Em que $E_{v}$ é a taxa de evasão, $E_{p}$ é o número de alunos que evadiram no período analisado e $M_{p}$ o número de alunos que se matricularam no período analisado. 


\begin{tabular}{ccccccccc}
\hline Curso & \multicolumn{2}{c}{$\begin{array}{c}\text { Desistência } \\
\text { Oficial (\%) }\end{array}$} & \multicolumn{2}{c}{$\begin{array}{c}\text { Transferência } \\
\text { Interna (\%) }\end{array}$} & \multicolumn{2}{c}{$\begin{array}{c}\text { Abandono } \\
\text { (\%) }\end{array}$} & \multicolumn{2}{c}{$\begin{array}{c}\text { Jubilamento } \\
(\%)\end{array}$} \\
\hline & Homens & Mulheres & Homens & Mulheres & Homens & Mulheres & Homens & Mulheres \\
\hline $\begin{array}{c}\text { Engenharia } \\
\text { Mecânica }\end{array}$ & 64,70 & 74,34 & 14,03 & 7,69 & 16,74 & 12,82 & 4,52 & 5,13 \\
\hline $\begin{array}{c}\text { Engenharia } \\
\text { Mecatrônica }\end{array}$ & 60,00 & 83,33 & 15,46 & 5,56 & 13,64 & 0,00 & 10,91 & 11,11 \\
\hline $\begin{array}{c}\text { Engenharia } \\
\text { Aeronáutica }\end{array}$ & 72,83 & 68,97 & 7,61 & 10,34 & 13,04 & 20,69 & 6,50 & 0,00 \\
\hline Média & 65,83 & 75,55 & 12,37 & 7,86 & 14,47 & 11,17 & 7,31 & 5,41 \\
\hline
\end{tabular}

Também foi possível determinar o número de egressos, por gênero e por curso, para os diferentes tipos de evasão considerados (desistência oficial, transferência interna, abandono e jubilamento).

\subsection{Análise de dados}

A partir dos dados e dos procedimentos citados anteriormente, determinou-se que apenas $14 \%$ de todos os discentes matriculados nos cursos de Engenharia Mecânica, Mecatrônica e Aeronáutica, entre os anos de 2010 e 2019, eram do sexo feminino. Também constatou-se que, no mesmo período destacado anteriormente, 509 pessoas evadiram, sendo 86 do sexo feminino, o que representa uma porcentagem de $17 \%$.

Quanto as taxas de evasão, nos três cursos analisados, as mulheres evadiram mais do que os homens no período entre 2010 e 2019.

Tabela 2 - Taxas de evasão para o período 2010-2019.

\begin{tabular}{lll}
\hline Curso & \multicolumn{2}{l}{ Taxa de evasão (\%) } \\
\hline & Homens & Mulheres \\
\hline Engenharia Mecânica & 24,47 & 32,77 \\
\hline Engenharia Mecatrônica & 23,34 & 28,57 \\
\hline Engenharia Aeronáutica & 24,47 & 27,62 \\
\hline
\end{tabular}

Fonte: Elaborada pelo autor.

A taxa de evasão média dos homens para os cursos de Engenharia Mecânica, Mecatrônica e Aeronáutica foi de aproximadamente $24 \%$, enquanto a taxa de evasão das mulheres foi de $30 \%$.

Em relação aos tipos de evasão, é possível perceber que homens e mulheres evadem mais a partir da desistência oficial. Percentualmente, a evasão de homens por transferência interna, abandono e jubilamento é maior do que das mulheres, o inverso acontece para a desistência oficial.

Tabela 3 - Tipos de evasão, por gênero e por curso para o período 2010-2019.

Fonte: Elaborado pelo autor.

\section{DISCUSSÃO}

A partir das análises apresentadas é possível perceber que, em concordância com a literatura, as mulheres ainda são sub-representadas em cursos de STEM, especificamente para o cenário da pesquisa, nos cursos de Engenharia Mecânica, 
Mecatrônica e Aeronáutica da Universidade Federal de Uberlândia. A situação torna-se ainda mais alarmante quando analisamos as taxas de evasão. Considerando o número de ingressantes e a taxa de evasão, é possível estimar que, em média, apenas 20 mulheres se formam por ano nos três cursos, em oposição aos 130 homens.

Os resultados obtidos em relação a taxa de evasão por gênero em cursos de Engenharia estão em concordância com os apresentados em pesquisas relacionadas. Isphording e Qendrai (2019) em ampla pesquisa desenvolvida com a colaboração de Universidades da Alemanha também concluíram que mulheres evadem mais que homens em cursos relacionados à ciência, tecnologia, engenharia e matemática.

É importante destacar que a quantidade de ingressantes do sexo feminino, entre o período de 2010 a 2019, sofreu alterações, como mostrado na Figura 1.

Figura 1: Número de matriculados, por gênero e por ano, para os três cursos.

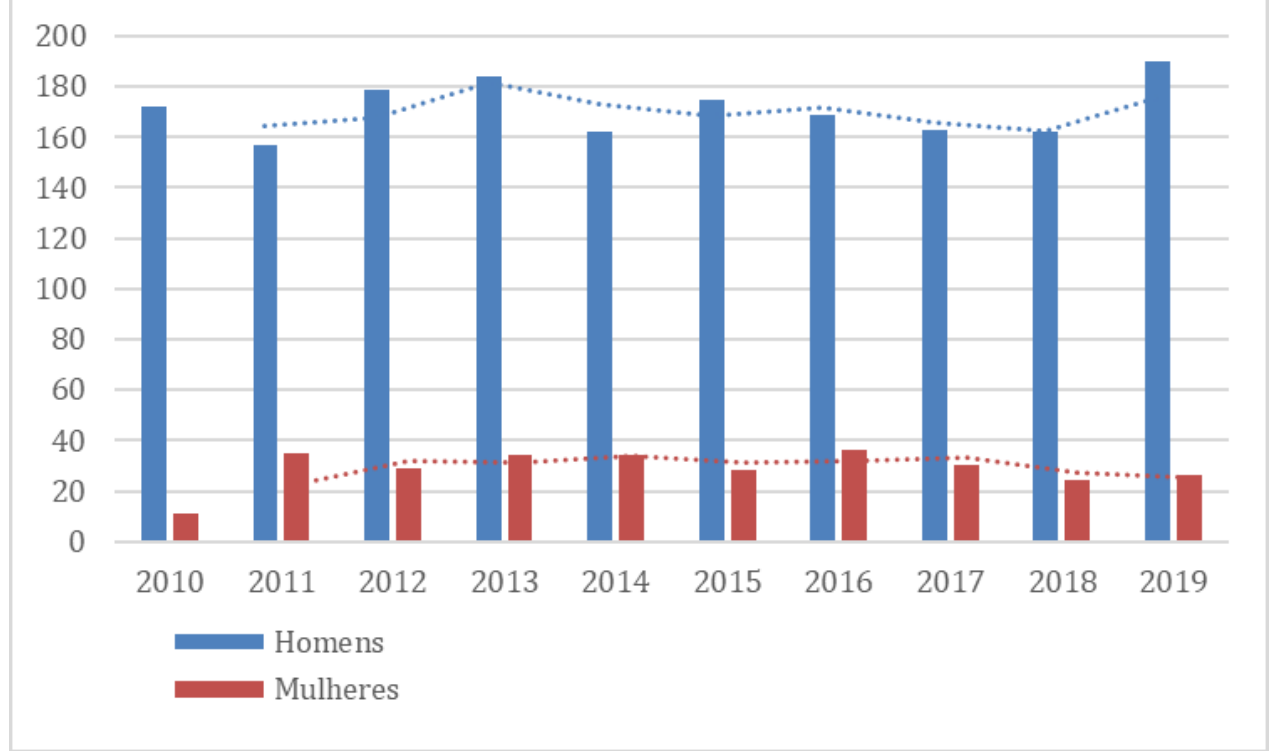

Fonte: Elaborada pelo autor.

Acompanhando a linha de tendência do gráfico, observa-se que no ano de 2010, o número de mulheres atingiu seu mínimo, com apenas 11 matriculadas nos 3 cursos. Entre o ano de 2010 e 2015, há uma tendência de crescimento do número de mulheres, atingindo um máximo de 35 nesse período. Entre 2015 e 2019, observamos uma tendência de diminuição do número de mulheres, atingindo um mínimo de 24 em 2018.

O comportamento apresentado na Figura 1 contrapõe os dados divulgados pelo IBGE (Instituto Brasileiro de Geografia e Estatística) e INEP, os quais indicam uma ampliação da participação das mulheres em carreiras predominantemente masculinas e uma mudança no panorama.

\section{CONSIDERAÇÕES FINAIS}

Primeiramente, retornando aos objetivos do presente trabalho, concluímos que foi possível a validação da hipótese inicialmente levantada de que, nos cursos de engenharia, as mulheres evadem mais que os homens. Mais do que isso, as informações aqui levantadas mostram a importância do desenvolvimento e da continuidade de estudos sobre evasão, mulheres em carreiras STEM e diversidade de gênero. Mostram também que há 
ainda um longo caminho a ser percorrido, tratando-se da modificação do Ensino Superior no Brasil de maneira a criar ambientes mais inclusivos e acolhedores, onde mulheres sintam-se pertencentes e em segurança e, onde todos possam receber o apoio que tanto necessitam.

Para tanto, os autores do presente trabalho estão desenvolvendo um estudo sobre os motivos que levaram à evasão de homens e mulheres e suas diferenças, de maneira a gerar propostas que possam contribuir para a transformação de tal realidade.

Sugere-se uma ampliação do estudo para outros cursos de engenharia e para outras Instituições de Ensino Superior no Brasil de maneira a gerar mais objetos de comparação, para que sugestões gerais possam ser feitas, assim como sugestões específicas para cada Instituição e/ou curso.

Sugere-se ainda um maior acompanhamento dos discentes, desde o momento da matrícula até o momento em que os mesmos deixam a instituição, realizando entrevistas ao longo da graduação e durante a saída, seja por evasão ou por conclusão do curso, com o objetivo de entender mais sobre as vivências do aluno na Instituição de Ensino, além de torná-lo parte dos processos de melhoria, através de possíveis sugestões.

Tais propostas buscam, dessa forma, solucionar o problema da evasão universitária, uma questão não só educacional, como econômica e política e, da baixa quantidade de mulheres atuando em carreiras STEM, um reflexo da sociedade patriarcal, preconceituosa e discriminatória.

\section{REFERÊNCIAS}

ABBAD, Gardênia; CARVALHO, Renata Silveira; ZERBINI, Thaís. Evasão em curso via internet: explorando variáveis explicativas. RAE- eletrônica, São Paulo, v. 5, n. 2, Art. 17, p. 01-26, jul./dez. 2006.

BOUND, John; LOVENHEIM, Michael e TURNER, Sarah. Why have college completion rates declined? An analysis of changing student preparation and collegiate resources. 2009.

COMISSÃO ESPECIAL DE ESTUDOS SOBRE EVASÃO (MEC/ANDIFES / ABRUEM/ SESU). Diplomação, retenção e evasão nos cursos de graduação em instituições de ensino superior públicas. Brasília, 1996.

GONZALEZ, Heather B.; KUENZI, Jeffrey J. Science, technology, engineering and mathematics (STEM) education: a primer. Congressional Research Service, August, 2012.

GUEDES, R. S.; MONTENEGRO, R. D. As estudantes de Engenharia: do Sonho ao Tormento Acadêmico. Paraíba, 2018.

ISPHORDING, I. E; QENDRAI, Pamela. Gender differences in student dropout in STEM. Germany, 2019.

LIMA, Michelle Pinto. As mulheres na Ciência da Computação. Revista Estudos Feministas. Florianópolis, v. 21, n. 3, p. 793-816, set./dez., 2013. 
MELO, Geovana; NAVES, Marisa. Retenção e evasão: desafios para a gestão da educação superior. Uberlândia, 2016.

OLIVEIRA, R.E.C. Vivências acadêmicas: interferências na adaptação, permanência e desempenho de graduandos de cursos de engenharia de uma instituição pública federal. Marília, 2015.

PALHARINI, F. A. Evasão, exclusão e gestão acadêmica na UFF: passado, presente e futuro. Cadernos do ICHF: Série Estudos e Pesquisas. Universidade Federal Fluminense - Instituto de Ciências Humanas e Filosóficas. Niterói, 2010.

PEREIRA, J. T. V. Uma contribuição para o entendimento da evasão. Um estudo de caso: Unicamp. Revista da avaliação da educação superior, v. 1, n. 2. São Paulo, 1996.

PEREIRA, A. C. F; FAVARO, N. A. L. G. História da mulher no ensino superior é suas condições atuais de acesso e permanência. Paranavaí, 2017.

PINTO, E. J. S; AMORIM, V.G. Gênero é educação superior: um estudo sobre as mulheres na física. Paraíba, 2015.

PRESTES, E. M. Da T.; FIALHO, M. G. D.; PFEIFFER, D. K. A evasão no ensino superior globalizado e suas repercussões na gestão universitária. João Pessoa, 2014.

RICOLDI, Arlene; ARTES, Amelia. Mulheres no ensino superior brasileiro: espaço garantido e novos desafios. São Paulo, 2016.

SILVA FILHO, R. L. L.; MOTEJUNAS, P. R.; HIPÓLITO, O.; LOBO, M. B. de C. M. A evasão no ensino superior brasileiro. Cadernos de Pesquisa, v. 37, n. 132, p. 641-659, set./dez, 2007.

SILVA, G. P. Análise de Evasão no Ensino Superior: uma proposta de diagnóstico de seus determinantes. 2013.

SMITH, Jeremy; NAYLOR, Robin. Dropping Out of University: A Statistical Analysis of the Probability of Withdrawal for UK University Students.2011.

STINEBRICKNER, Ralph; STINEBRICKNER, Todd. A Major in Science? Initial Beliefs and Final Outcomes for College Major and Dropout. 2013.

UNESCO. Decifrar o código: educação de meninas e mulheres em ciências, tecnologia, engenharia e matemática (STEM). 2018.

VALDIEIRO, A. P.; SANTOS, C. P.; RAIA, L. A.; DREWS, S. B. T.; THESING, N. J.; NHERING, C. M.; Análise da evasão discente no curso de engenharia mecânica da UNIJUÍ e proposição de soluções. Juiz de Fora, 2014.

\section{GENDER DIVERSITY AND UNIVERSITY DROPOUT IN ENGINEERING COURSES}


Abstract: The present study seeks to understand and quantify the phenomenon of evasion in higher education institutions, more specifically, the differences between the evasion of women and men in the courses of Mechanical Engineering, Mechatronic Engineering and Aeronautical Engineering at the Federal University of Uberlândia, through validation the hypothesis that women have higher dropout rates in engineering courses, taking into account the diversity of the human factor and the difficulties faced by women who choose to pursue STEM careers (Science, Technology, Engineering and Mathematics). The data were collected from statistical reports from the Educational Institution for the Mechanical, Mechatronic and Aeronautical Engineering courses at the Federal University of Uberlândia between the years 2010 to 2019. The results obtained from qualitative data analysis point to greater evasion of female students. While only $24.17 \%$ of men enrolled in the three courses dropped out, $29.65 \%$ of women did so.

Keywords: Dropout. STEM. Engineer. Gender. 